\title{
Fecal Transplant- Fecal Enema
}

Shivani Modi

American International Medical University, Saint Lucia. Email: shvnmd@gmail.com

DOI: http://doi.org/10.38177/AJBSR.2020.2402

Copyright: (92020 Shivani Modi. This is an open access article distributed under the terms of the Creative Commons Attribution License, which permits unrestricted use, distribution, and reproduction in any medium, provided the original author and source are credited.

\section{ABSTRACT}

Fecal enema is when the doctor transplants feces from the healthy individual into another individual in-order to restore the normal balance of bacteria in the gut. Fecal enema is also known as fecal bacteriotherapy or Fecal microbial transplant (FMT). Although the concept sounds unpleasant, it is important to know that our gut requires bacteria in-order to digest the food and function efficiently, but some medical conditions and antibiotics can destroy these good bacteria in the gut. Once the bacteria are lost it is very difficult to restore it back. A fecal transplant is one way to reintroduce them. Because the stool is about 50\% bacteria, the theory behind the transplant is that it can replace bacteria that have gone missing from the gut $[2,4,12,16]$. Fecal transplants began in old Chinese prescription over 1,700 years prior. Previously, this system included drinking a fluid suspension of someone else's excrement - a profoundly dangerous procedure. The present fecal transplants are sterile and safe, and there is a developing assortment of research to help their utilization [4].This article focusses on what is fecal transplant, benefits vs risks, different methods of fecal transplant and possible candidates for fecal enema.

Keywords: Colonoscopy, Crohn's Disease, Enema, Inflammatory bowel disease.

\section{What is Fecal Transplant?}

Fecal transplantation is executed as a treatment for monotonous $C$. difficile colitis.

C. difficile colitis, a complication of antibiotic treatment, may be connected with diarrhea, stomach cramping and so often fever. If individual is past 65 years of age or possibly have wearisome sicknesses or chronic diseases, patient may be at higher danger for having severe illnesses. Investigation relies upon a stool DNA test that distinguishes the organism or bacteria. ${ }^{[2,3,12]}$

Patient detected with C. difficile infection, the doctor will treat the basic infection with antibiotics that expressly targets around the $\mathrm{C}$. difficile organism. The antibiotics used for the treatment include metronidazole, vancomycin and fidaxomycin. In 30 percent of treated individuals, the infection returns back in two, three days or weeks after finishing the antibiotic course. The doctor may treat this first relapse with another round of hostile to disease treatment. Regardless, in those individuals who continue having continuous C. difficile colitis, fecal transplantation is another option. Doctors screen fecal transplant donors to guarantee that their gut and excrement are solid and disease free. They will test them for different maladies, for example, hepatitis. ${ }^{[2,4,6]}$

Much of the time, a specialist will transfer the feces to the beneficiary through a colonoscope. A colonoscope is a little, adaptable cylinder that they can embed into the colon through the rectum. Individuals are normally given narcotic medications before the procedure, so they won't feel any pain or uneasiness.

Another procedure includes infusing fluid feces by means of a bowel enema as opposed to utilizing a colonoscope. An enema is a liquid laxative that is administered rectally. ${ }^{[4,5,7]}$

Although this method is used less frequently and there is not much data about the efficiency of this method. A recent report published in 2013 in the New England Journal of Medicine showed that fecal transplantation is more 
fruitful than oral vancomycin in hindering further relapses in individuals who have recently had recurrent $\mathrm{C}$. difficile colitis. ${ }^{[4,16]}$

\section{Uses}

In the United States, C. difficile colitis kills about 15,000 people each year, often due to severe colon inflammation. In a small scale study of 2014 preliminary, $70 \%$ of the members had no side effects after one fecal transplant treatment. The general success rate was $90 \%$ among the individuals who underwent multiple fecal transplant. The members likewise had less defecations problems and stated their general wellbeing more exceptionally following treatment. Different examinations have detailed comparative achievement rates. Specialists have discovered that fecal transplants may help treat IBD, however their adequacy differs. ${ }^{[2,4,16]}$

Doctors may likewise prescribe fecal transplants to other gastrointestinal conditions. For example, issues in patients with chronic Inflammatory bowel disease (IBD). Fecal transplants may likewise help with Inflammatory bowel syndrome (IBS), an inadequately comprehended condition that causes a wide range of stomach related issues. ${ }^{[4,8,10]}$

\section{Fecal Transplant research}

A 2016 survey found that achievement rates in preliminaries ran from $36.2 \%$ to $77.8 \%$, indicating a requirement for further research. ${ }^{[4]}$

Research on the more broad effects of gut microorganisms is now developing. Fecal transplants could treat a combination of conditions which is mentioned in the article of a 2016 review, for instance,

a) Diabetes

b) Incessant weakness disorder

c) Fibromyalgia

d) Obesity

e) Hay fever

f) Arthritis

g) Asthma

Although, mechanism by which fecal transplant helps treat these diseases is still under research.

\section{Who could be a potential donor?}

Identifying a potential donor prior to your fecal transplantation is also an important key factor along with the procedure. Screening the donors for any infectious pathogens such as Hepatitis A, B and C, HIV and any ova and parasites. ${ }^{[4,9,13]}$ Donors should not:

- Have had any tattooing or body piercing in past six months

- Have any history of incarceration

- Have any history of drug use 
- Have any history of high-risk sexual behavior

- Have any chronic GI disorders, such as inflammatory bowel disease

- Have had any antibiotic exposure in the past six months

- Be immunocompromised

\section{Who shouldn't have a fecal transplant?}

Fecal transplants aren't recommended for people who are immunocompromised because of:

a. HIV

b. Immunosuppressants

c. cirrhosis

d. a recent bone marrow transplant ${ }^{[13,14]}$

\section{Procedure}

Fecal Transplant is usually performed by colonoscopy or by enema. Feces are obtained from the donor six hours prior to the fecal transplant. Sample is further checked for any ova and parasites. If the sample is appropriate for transplant it is then made ready for the procedure by adding sterile water and filtering it until solids are removed. The transplant is infused in the recipient via colonoscopy or by enema.

Before the procedure, patient should be given instructions to:

a) Patient needs to stop taking antibiotics two days prior to the procedure.

b) Stop eating solid foods after liquid laxative or previous night before the procedure.

c) Let the doctor know about any allergy.

Patient will be given anesthesia to avoid pain and discomfort. When the procedure is done through a colonoscope, the feces can be spread all through the colon, which is progressively convincing. A proton-pump inhibitor is given all the while in order to shield the beneficial bacteria from being killed by stomach acids.

During the procedure, a colonoscope is inserted from the rectum to the larger end of the intestine.

The extension twists, so the specialist can move it around the bends of the colon. Patient might be approached to change position every so often to enable the specialist to move the degree. The extension additionally blows air into your colon, which grows the colon and enables the specialist to see all the more clearly. ${ }^{[2,4,12,14,16]}$

If the enema method is used, the feces test is put into the enema bag or bottle, then infused into the rectum where it is held as long as possible. This is done more than once through the span of 5 to 10 days, directly introducing the normal flora back into the intestinal tract.

Fecal transplants given by enema are typically less invasive and lower in cost than colonoscopies. ${ }^{[4,5,13]}$ 

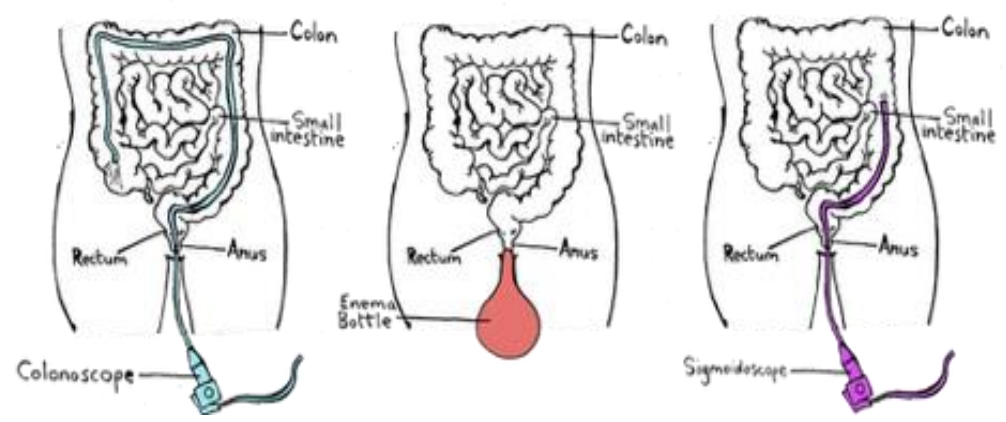

Figure 1. Procedures for fecal Transplant ${ }^{[1]}$

\section{After Surgery}

Fecal transplants are usually outpatient procedure, where patient can go home the same day. Most people experience relief of GI symptoms within 24-72 hours but for the gut to resemble like the donor it can take unto 3-4 months. ${ }^{[12]}$

\subsection{Risks and Complications}

Following a fecal transplant, you may experience a few side effects, including:

1. Abdominal discomfort or cramping

2. Constipation

3. Bloating

4. Diarrhea

5. Belching or flatulence

Contact your healthcare provider right away if the pain becomes severe or you also experience:

- severe abdominal swelling ${ }^{[12,15,16]}$

○ vomiting

- blood in your stool

\subsection{Benefits}

As indicated by the American College of Gastroenterology, 5 to 15 percent of grown-ups — and 84.4 percent of babies and healthy newborn children - have an ordinary measure of $\mathrm{C}$. diff in their digestion tracts. It doesn't cause issues and aides in keeping up the typical bacterial populace of the gut. Other microorganisms in your digestion tracts normally keep the number of inhabitants in C. diff under tight restraints, keeping it from causing a contamination. A fecal transplant can reintroduce these microbes into your GI tract, enabling them to anticipate future excess of C. diff.

Out of nine studies found that fecal transplants improved IBS manifestations in 58 percent of members. But, the nine investigations were different in their criteria, structure, and examination. Four preliminaries were reviewed, contrasting Ulcerative Colitis reduction rates in individuals that had gotten a fecal transplant versus a fake 
treatment. The individuals who got a fecal transplant had a reduction rate of 25 percent, contrasted with 5 percent for those in the fake treatment gathering. An ongoing study in mice included two gatherings: one sustained a high-fat diet and another encouraged an ordinary fat eating routine and set on an activity routine. The mice on the high-fat eating regimen got fecal transplants from the mice in the subsequent gathering. This seemed to reduced inflammation and improves digestion. They even distinguished a few organisms related with these impacts, however its vague how these outcomes will decipher in people. ${ }^{[16,17,4]}$

While some of these results are promising, there's still a big need for more research in this area to determine the effectiveness and safety of fecal transplants for these uses.

\subsection{FDA Standpoint}

June 13, 2019, after 2 immunocompromised patients experienced adverse effect, contracted drug-resistant infections, according to a statement from the agency's website. The US Food and Drug Administration issued a safety alert for fecal transplant procedures. The FDA warns that fecal matter should be tested for drug-resistant bacteria. ${ }^{[15-21]}$

\section{What is DIY?}

Internet and social media provides people with so much information about fecal transplant. Although there is a new Do-It-Yourself (DIY) concept going around on social media and internet. Statistics says about 10,000 people yearly in United States perform DIY.

DIY is preferred by people to get around the regulations of FDA, which sounds like a good idea but it is not. ${ }^{[12,16-21]}$ Few reasons why DIY is unsafe are:

- Without proper donor screening, people may put themselves at risk of contracting any disease.

- Doctors performing fecal transplants have extensive training in how to safely make a stool preparation for transplant.

- The research into the long-term effects and safety of fecal transplants is still limited, particularly for conditions other than C. diff infection.

Clinicians and patients should know about the resources accessible through web based life and the Internet. It should be made known that a few sites fall outside directed clinical practice. Private facilities offering FMT need to guarantee that they are offering FMT inside a managed structure.

\section{Future of Fecal Transplant?}

Late studies show how FMT can be advantageous to kids who have explicit GI conditions, for example, Crohn's ailment or ulcerative colitis. Researchers are additionally beginning to test FMT for nut hypersensitivity, in light of proof that moving "good" microscopic organisms can rebalance the invulnerable framework.

FMT is an interesting issue among scientists and clinicians. ClinicalTrials.gov uncovers around 170 examinations on FMT, with applications as differing as type 2 diabetes, cirrhosis and HIV. Furthermore, in a clinical preliminary 
at Boston Children's Hospital, scientists are seeing in the case of giving shelled nut unfavorably susceptible individuals the "good" microbes from a non-hypersensitive individual avoids unfavorably susceptible responses. ${ }^{[12]}$

\section{Summary}

Accepting defecation from a healthy giver may appear to be particular, however the science supporting fecal transplants is turning out to be progressively standard. At the point when different medications come up short, this imaginative system may normally reestablish the gut's wellbeing, enabling useful microscopic organisms to develop and improve an individual's wellbeing. Fecal transplants are a promising potential treatment for a variety of conditions. Today, they are utilized essential to treat repeating C. diff contaminations.

There is a tremendous amount of data accessible about FMT through web-based social networking that has the potential for causing harm. A community approach between clinicians, general people, and controllers of social media life should help square potentially dangerous information of data via web-based networking media stages.

\footnotetext{
References

[1] https://mosaicscience.com/sites/default/files/styles/story_999/public/Poo2_0.png?itok=HWg01X7p

[2] https://www.hopkinsmedicine.org/gastroenterology_hepatology/clinical_services/advanced_endoscopy/fecal_transplantation.html

[3] https://www.webmd.com/digestive-disorders/news/20151209/diy-fecal-transplant\#1

[4] https://www.medicalnewstoday.com/articles/325128.php

[5] https://www.openbiome.org/stool-donation

[6] https://ubiome.com/blog/post/heck-fecal-transplant/

[7] https://www.exmed.net/blog/expressmedicalsupply/post/2018/08/29/how-long-does-it-take-an-enema-to-work.aspx

[8] https://www.exmed.net/blog/expressmedicalsupply/post/2018/08/29/how-long-does-it-take-an-enema-to-work.aspx

[9] https://www.ncbi.nlm.nih.gov/pubmed/13592638

[10] https://www.nih.gov/news-events/news-releases/clinical-trial-testing-fecal-microbiota-transplant-recurrent-diarrheal-disease-begins

[11] http://www.childrenshospital.org/conditions-and-treatments/treatments/fmt

[12] https://www.healthline.com/health/fecal-transplants-the-key-to-improving-gut-health\#diy

[13] https://www.ncbi.nlm.nih.gov/pmc/articles/PMC5840108/

[14] https://www.webmd.com/colorectal-cancer/colonoscopy-what-you-need-to-know\#1

[15] https://www.fda.gov/vaccines-blood-biologics/safety-availability-biologics/important-safety-alert-regarding-use-fecal-microbiota-tra nsplantation-and-risk-serious-adverse

[16] https://www.ncbi.nlm.nih.gov/pmc/articles/PMC5935081/

[17] https://www.sciencedaily.com/releases/2019/01/190121103408.htm

[18] https://www.webmd.com/digestive-disorders/news/20190614/fda--infections-1-death-after-fecal-transplants\#1

[19] https://www.the-scientist.com/news-opinion/fda-suspends-clinical-trials-involving-fecal-transplants-66009

[20] https://www.mdedge.com/familymedicine/article/166780/gastroenterology/how-effective-and-safe-fecal-microbial-transplant

[21] https://www.theatlantic.com/health/archive/2019/06/the-uncertain-future-of-fecal-transplants/592363/
} 